\title{
Contact Interactions and Resonance-Like Physics at Present and Future Colliders from Unparticles * ${ }^{*}$
}

\author{
Thomas G. Rizzo \\ Stanford Linear Accelerator Center, 2575 Sand Hill Rd., Menlo Park, CA, 94025
}

\begin{abstract}
High scale conformal physics can lead to unusual unparticle stuff at our low energies. In this paper we discuss how the exchange of unparticles between Standard Model fields can lead to new contact interaction physics as well as a pseudoresonance-like structure, an unresonance, that might be observable at the Tevatron or LHC in the Drell-Yan channel. The specific signatures of this scenario are quite unique and can be used to easily identify this new physics given sufficient integrated luminosity.
\end{abstract}

Submitted to Journal of High Energy Physics (JHEP)

${ }^{*}$ Work supported in part by the Department of Energy, Contract DE-AC02-76SF00515

†e-mail: rizzo@slac.stanford.edu 
In a recent paper, Georgi[1] has speculated on the existence of a high scale conformal sector[2] which may couple to the various gauge and matter fields of the Standard Model(SM). This new sector can lead to important phenomenological consequences in our low energy world through the interactions of some new 'stuff', termed unparticles, whose properties have begun to be explored in an ever increasing number of phenomenological analyses $[3,4,5,6,7,8,9,10,11,12$, $13,14,15,16,17,18,19,20,21,22,23,24,25]$. There are good reasons to believe that the physics of such unparticles may best be explored at TeV scale colliders. Signals at such machines can result either from unparticle emission in otherwise SM processes, which leads to a new source of missing energy, and/or their exchange between SM fields which leads to new contact-like interactions. Extending earlier work $[3,4,21,25]$, in this paper we will explore how the exchange of unparticles between SM fields can be probed in Drell-Yan collisions at the Tevatron and LHC as well as at ILC. The well-understood Drell-Yan channel is a particularly clean one at hadron colliders and provides us with an almost background free laboratory to look for many kinds of new Terascale interactions. The breaking of the conformal symmetry near the electroweak scale through the SM Higgs vev[15] leads to a pseudoresonance-like structure at, e.g., the LHC, that we term an unresonance, which can be used to uniquely identify unparticles as source of the new physics.

As discussed in the literature[19], there are very many ways for unparticles to interact with SM fields and the particular choice of a subset of these to examine will depend on a number of assumptions. For example, the interaction of a spin-1 unparticle with a pair of ordinary, in principle different, fermions may be written as

$$
\frac{1}{\Lambda^{d-1}} \bar{f} \gamma_{\mu}\left(c_{L} P_{L}+c_{R} P_{R}\right) \tilde{f} \mathcal{O}^{\mu}
$$

where $\Lambda$ is an effective mass scale, $d$ is the non-canonical scaling dimension of the unparticle field, which is expected to lie in the range $1 \leq d \leq 3$, and $c_{L, R}$ are assumed to be $O(1)$ coefficients; $P_{L, R}=\left(1 \mp \gamma_{5}\right) / 2$ are the helicity projection operators as usual. Recall that a free ordinary gauge field has $d=1$ leading to a dimensionless coupling. For a spin-0 unparticle on the otherhand, the 
corresponding helicity-preserving interaction takes the form[1]

$$
\frac{1}{\Lambda^{d-2}} \bar{f} \gamma_{\mu}\left(c_{L}^{\prime} P_{L}+c_{R}^{\prime} P_{R}\right) \tilde{f} \partial^{\mu} \mathcal{O}
$$

Such an operator is relatively suppressed for two reasons: $(i)$ due to the presence of the derivative an additional power of $\Lambda$ appears in the denominator and, more importantly, (ii) the mass dimension compensation in the numerator is through a linear combination of the $f, \tilde{f}$ masses. Since the initial state fermions at both the hadron colliders and ILC are all quite light it will be essentially impossible to probe such SM-fermion unparticle scalar interactions at these machines. Thus we will only consider the spin-1 unparticle case in the discussion below.

At the Tevatron and LHC, gluon initial states may also contribute in the Drell-Yan channel[21] provided that they also couple to unparticles through an operator of the form

$$
\sim \frac{1}{\Lambda^{d+1}} G_{\mu \alpha}^{a} G_{a, \nu}^{\alpha} \mathcal{O}^{\mu \nu}
$$

where the 'field strength' $\mathcal{O}^{\mu \nu}=\partial^{\mu} \mathcal{O}^{\nu}-\partial^{\nu} \mathcal{O}^{\mu}$. However, in comparison to the coupling to fermion pairs, this interaction appears to be suppressed by two additional powers of $\Lambda$. Furthermore, this new interaction introduces another free parameter into the model describing the assumed relative strength of unparticle couplings to fermion and to gauge fields. We will briefly address these issues in the discussion below noting that for equal strength couplings this $g g$ contribution to Drell-Yan production is relatively suppressed in comparison to that arising from the $q \bar{q}$ initial state at both the Tevatron and LHC.

In what follows we will make some further assumptions about the nature of the unparticles and their interactions. Being SM singlets, the simplest possibility is to imagine that they couple universally in a flavor-blind manner to all of the SM fermion representations. In such a case all of the strong constraints that may arise from flavor-changing processes can be trivially avoided as they are naturally absent. This also implies that the couplings of the SM fermions to unparticles is purely vector-like and thus we can absorb the parameter combination $c_{L}+c_{R}$ above into the 
definition of $\Lambda$ for convenience. Furthermore, the unparticle coupling to the SM Higgs doublet, as discussed in Ref.[15], leads to a breakdown of conformal invariance once the Higgs obtains a vev. In such a situation, the authors of Ref.[15] note that the propagator for the unparticle will be modified and in a simple toy model will behave as

$$
\sim A_{d} \theta\left(P^{0}\right) \theta\left(P^{2}-\mu^{2}\right)\left(P^{2}-\mu^{2}\right)^{d-2} e^{i \pi(d-2)}
$$

with $A_{d}$ being the numerical factor given by Georgi[1]. We will assume that this form provides a reasonable description in this situation and we will discuss the implications of this form further below as it leads to striking collider signatures; we imagine, however, that the $\theta$ function threshold is somewhat smoothed out in a more realistic scenario but also that the turn-on of unparticle exchange remains very rapid. Note that for the processes of interest to us we do not need to assume that the unparticle is transverse, i.e., $\partial_{\nu} \mathcal{O}^{\nu}=0$, since the external fermions can be treated as massless to a very good approximation. In expression above $\mu$ is the effective 'low' energy scale where conformal invariance is broken in the unparticle sector. Note that this expression implies that the presence of $\mu \neq 0$, even if $\mu$ is only a few $\mathrm{GeV}$, cuts off the low-momentum unparticle exchange modes resulting in a very strong suppression of unparticle-induced contributions to many processes at low energies. These include, e.g., the contributions to $g-2$ for both $e$ and $\mu$, modifications to positronium decay as well as any of the remaining flavor-changing interactions involving $K, D$ and $B$ mesons. With such a non-zero $\mu$, the important bounds on the scale $\Lambda$ arising from these types of processes can be completely evaded ${ }^{\ddagger}$. It is also possible that even those, as yet unexplored, constraints arising from precision electroweak measurements can also be evaded for reasonable values of $\mu$ while simultaneously allowing values of $\Lambda$ to be not too far above $1 \mathrm{TeV}$. In that case unparticle physics may remain accessible at Tevatron, LHC and ILC energies. Perhaps one might naturally expect that $\mu \sim 1 \mathrm{TeV}$ and so is not too distant in magnitude from that of the SM Higgs vev, $246 \mathrm{GeV}$, which breaks the conformal symmetry in the SM; we will assume that this possibility is realized within an approximate order of magnitude in the analysis presented below. As we will

\footnotetext{
${ }^{\ddagger}$ These bounds, when $\mu=0$, can be particularly strong as shown in Fig. 1
} 
see the value of $\mu$ will play a very important role in collider searches for unparticle physics under the assumptions outlined above.

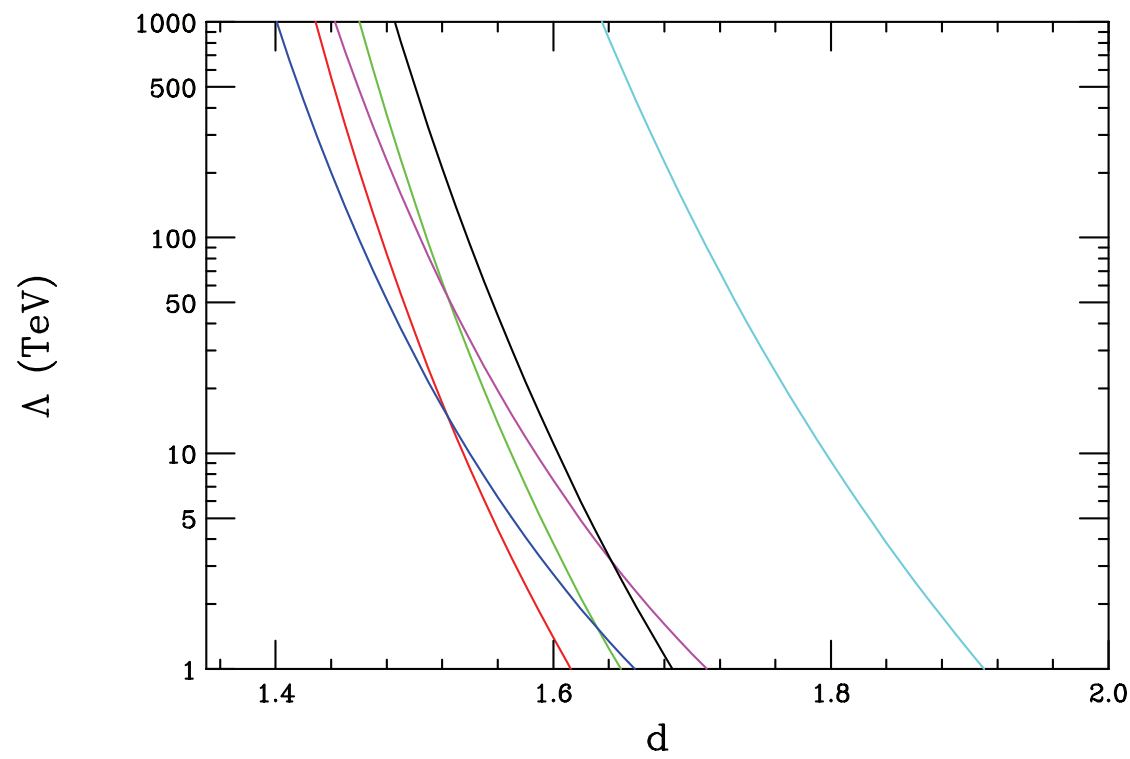

Figure 1: Low energy bounds of the scale $\Lambda$ as a function of $d$ from $(g-2)_{e}$ (red,green), $(g-$ $2)_{\mu}$ (blue,magenta) and orthopositronium decay(cyan,black). The first(second) member of each pair corresponds to purely vector(axial-vector) couplings. The excluded region is below and to the left of each curve.

Let us consider the contact-interaction-like contribution of unparticle exchange in the DrellYan process, $q \bar{q} \rightarrow \ell^{+} \ell^{-}$, as a function of the dilepton invariant mass, $M$. The parton-level triple differential cross section for this process can be easily calculated[4, 26]. Note that in addition to the usual SM terms, this subprocess differential cross section picks up an extra unparticle induced piece proportional to the following combination of factors for each initial state quark (neglecting terms odd in the scattering angle here which integrate to zero in the total cross section):

$$
2 f e^{2} Q_{e} Q_{q} \cos (d-2) \pi+2 f v_{e} v_{q} M^{2} \frac{\left(M^{2}-M_{Z}^{2}\right) \cos (d-2) \pi+M_{Z} \Gamma_{Z} \sin (d-2) \pi}{\left(M^{2}-M_{Z}^{2}\right)^{2}+\left(M_{Z} \Gamma_{Z}\right)^{2}}+f^{2},
$$

where $e^{2}=4 \pi \alpha, Q_{e, q}$ are the electron and quark charges and $v_{e, q}$ are the vector couplings of the electron and quarks to the SM $Z$; with $\hat{s}=M^{2}$, we have defined

$$
f=A_{d} \theta\left(\hat{s}-\mu^{2}\right) \frac{\hat{s}}{\Lambda^{2}}\left[\frac{\left(\hat{s}-\mu^{2}\right)}{\Lambda^{2}}\right]^{d-2},
$$


where $M_{Z}\left(\Gamma_{Z}\right)$ is the mass(width) of the SM $Z$. The factor $f$ arises directly from the assumed form of the unparticle propagator given above. Note that when $d=2$ we recover the ordinary, traditional dimension- 6 contact interaction apart from a normalization factor[29] and the $\theta$-function threshold. We now observe that for $\mu>0$ and $d<2$, if taken at face value, the factor $f$ leads to a very unusual threshold behavior for unparticle physics since $f$ vanishes when $\hat{s}<\mu^{2}$ but is very large when $\hat{s}$ is immediately above $\mu^{2}$. This means that a distorted peak will appear in the dilepton mass distribution in the region near $\hat{s}=\mu^{2}$ whose visibility will depend on the values of $d$ and $\Lambda$ as well as the detector mass resolution. When $d>2$ this unresonance structure vanishes and a more conventional contact-like interaction appears. In what follows we will concentrate on the more interesting range $1<d<2$.

As discussed above the unparticle coupling to both gluons and fermions simultaneously leads to an additional contribution to the Drell-Yan process which must be added incoherently to that arising from the $q \bar{q}$ initial state. Using the same normalization as in that case, the parton level cross section for $g g \rightarrow \ell^{+} \ell^{-}$is, apart from common overall factors, essentially given by

$$
\frac{3}{32} c^{2} f^{2}\left[\frac{\hat{s}}{\Lambda^{2}}\right]^{2}
$$

where $c$ describes the relative strength of the $g g$ coupling to unparticles relative to that of the corresponding flavor universal $q \bar{q}$ coupling one. Note the extra suppression by the additional powers of $\Lambda$ which we anticipated in the discussion above. Numerically, from a detailed calculation we then find that for $c=1$ and $\Lambda \geq M \geq 1 \mathrm{TeV}$ at the LHC, this $g g$ contribution is only $\sim 5-10 \%$ or less of that of the $q \bar{q}$ contribution to the total cross section; this relative contribution is even smaller at Tevatron energies since in that case both the $q$ and $\bar{q}$ are valence quarks. These results are found to be independent of the other unparticle model parameters $d$ and $\mu$. Thus the $g g$ contribution can generally be neglected in the discussion that follows to a very good approximation.

To match conditions at the Tevatron (LHC) as best as possible we employ approximate NNLO K-factors, apply rapidity cuts on the outgoing leptons, $\left|\eta_{\ell}\right|<1(2.5)$, and Gaussian smearing 
to the dilepton mass distribution with a $\sim 1 \%$ resolution to approximate that of, e.g., the CDF and ATLAS detectors for the electron final state[27, 28] in the relevant dilepton mass range. We can then compare the predictions of the SM with those of the unparticle scenario for different values of the parameters $d, \Lambda$ and $\mu$. The issue here is, of course, not just whether or not the effects of the unparticles are visible above the SM Drell-Yan background but whether the predictions for unparticle physics can be uniquely identified and also if the different values of the unparticle parameters are distinguishable at the Tevatron and LHC. Obtaining the value of $\mu$, given sufficient center of mass energy, will be the most straightforward of these measurements at any collider as it determines the unparticle threshold and the position of the corresponding resonance-like structure,

Figs. 2 and 3 show a selection of the predictions for this unparticle model at the Run II Tevatron. Here we see that the predictions of the SM are reproduced in all cases when $M<\mu$ but then a narrow peaking structure, the unresonance, is generated by the smeared singularity in the unparticle propagator. Note that there are no deviations from the SM until one sees the unresonance itself. This implies that indirect searches for this variety of unparticle physics are useless at values of $M$ below the resonance structure itself unlike, e.g., the indirect searches for more traditional resonances such as KK gravitons or new gauge bosons[26] which interfere with the SM in the sub-threshold mass regime. For example, at the ILC with a fixed center of mass energy no unparticle exchange signal would be observed unless $\mu<\sqrt{s}$ so that indirect constraints on the unparticle model parameters cannot be obtained using this quite standard technique. It is important to remember that although it generally appears as such, this unparticle structure is not an ordinary resonance like, e.g., a $Z^{\prime}$. This unresonance is an artifact of the propagator threshold and large $M$ behavior combined with the finite detector resolution and the rapid fall off of the parton densities.

It is clear from these figures that such a strange effect should be observable at the Tevatron for a reasonable range of the model parameters although the details of this structure are dependent on the specific choices made for the values of $d, \mu$ and $\Lambda$. The lack of observation of such a structure 

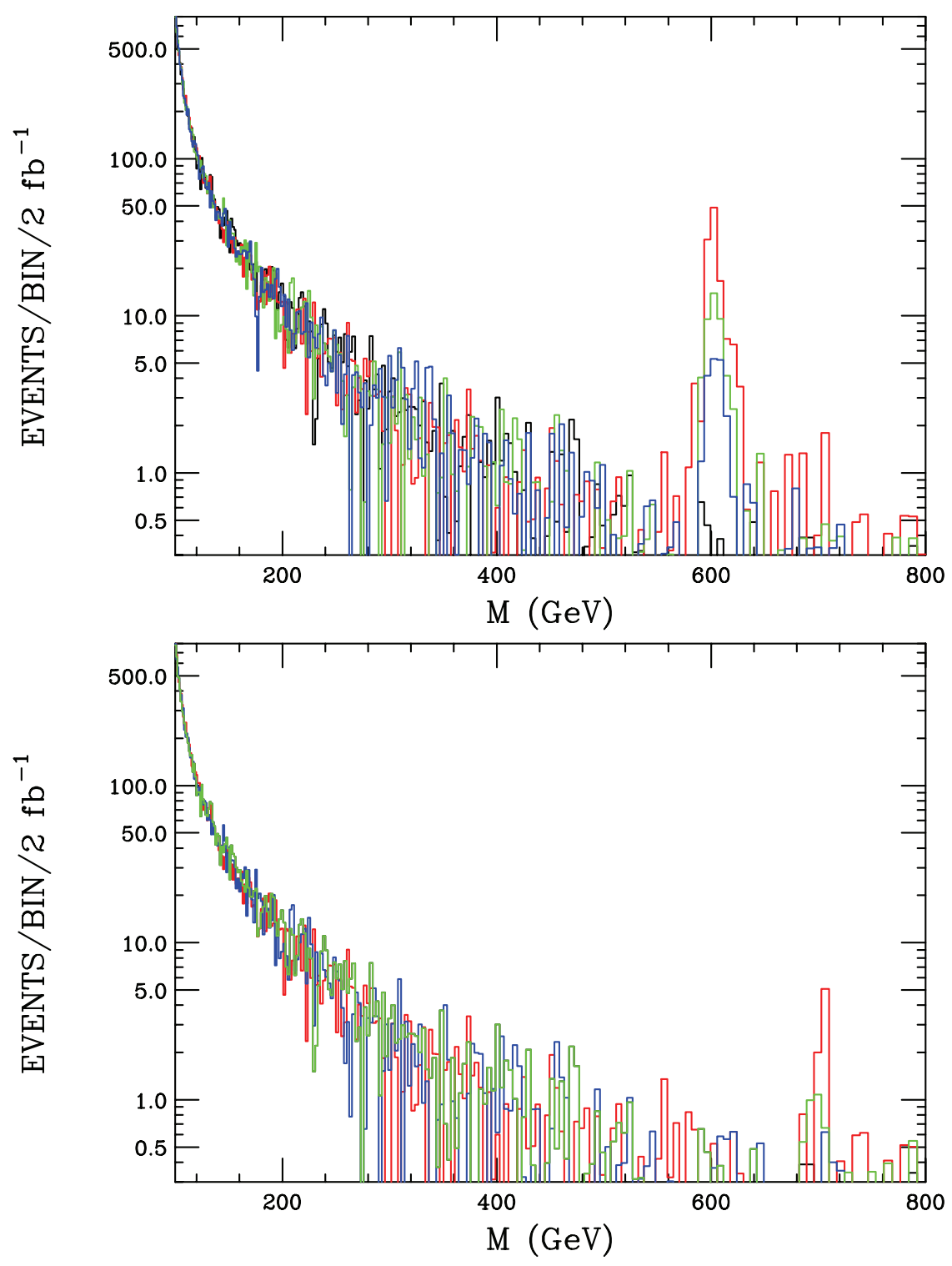

Figure 2: Drell-Yan dilepton mass distribution at the Tevatron assuming $\mu=600(700) \mathrm{GeV}$ with $d=1.5(1.6)$ in the top(bottom) panel. The red(green,blue) histogram corresponds to $\Lambda=1(2,3)$ $\mathrm{TeV}$, respectively. The almost invisible black histogram is the SM prediction. 

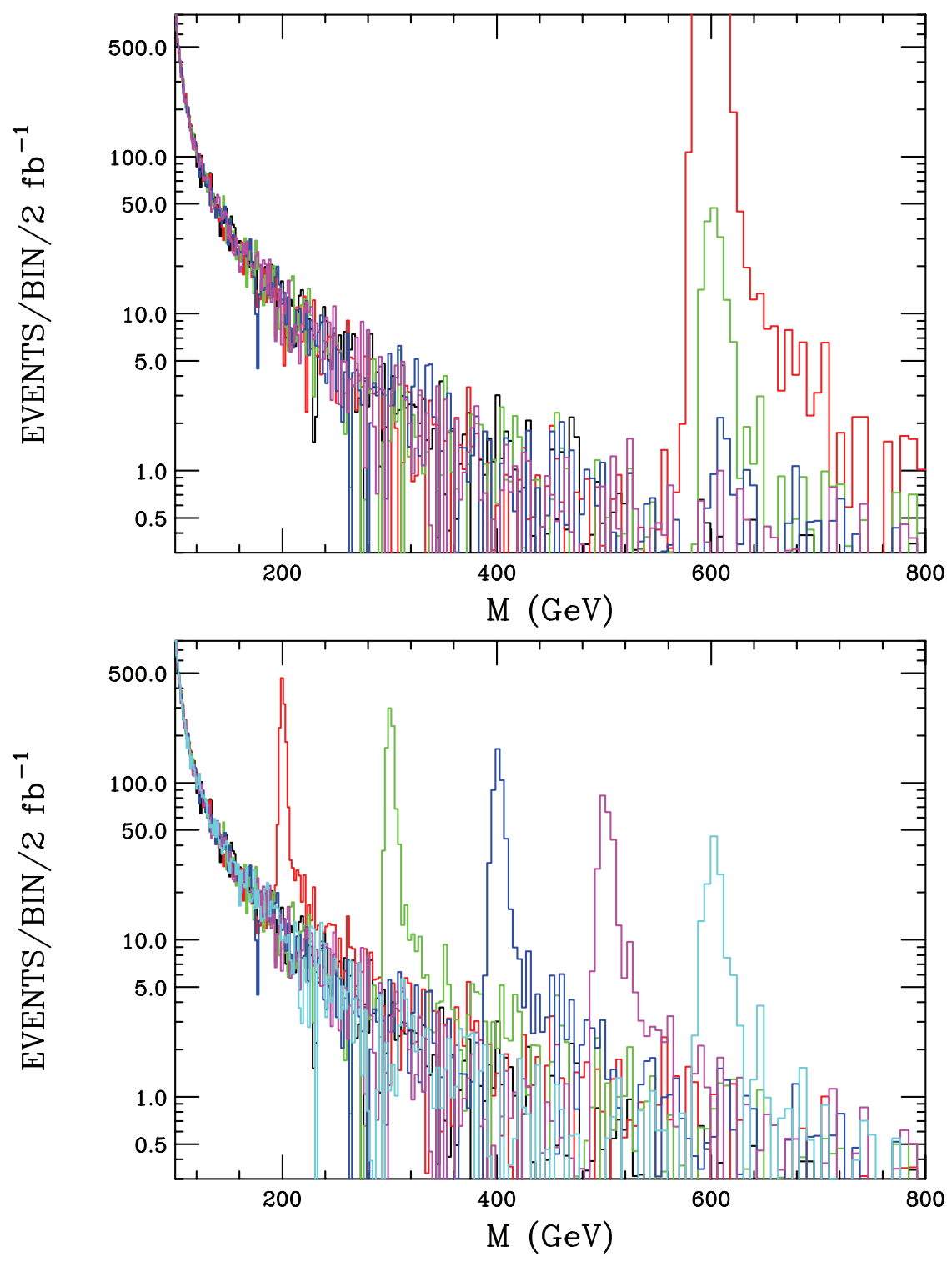

Figure 3: (Top) Same as the previous figure but now with $\Lambda=1 \mathrm{TeV}$ and $\mu=600 \mathrm{GeV}$ for $d=1.3(1.5,1.7,1.9)$ corresponding to the red(green,blue,magenta) histograms, respectively. (Bottom) In this case $\Lambda=1$ and $d=1.5$ with $\mu=200,300,400,500$ or $600 \mathrm{GeV}$. The SM prediction is the (almost invisible) black histogram in both panels. 


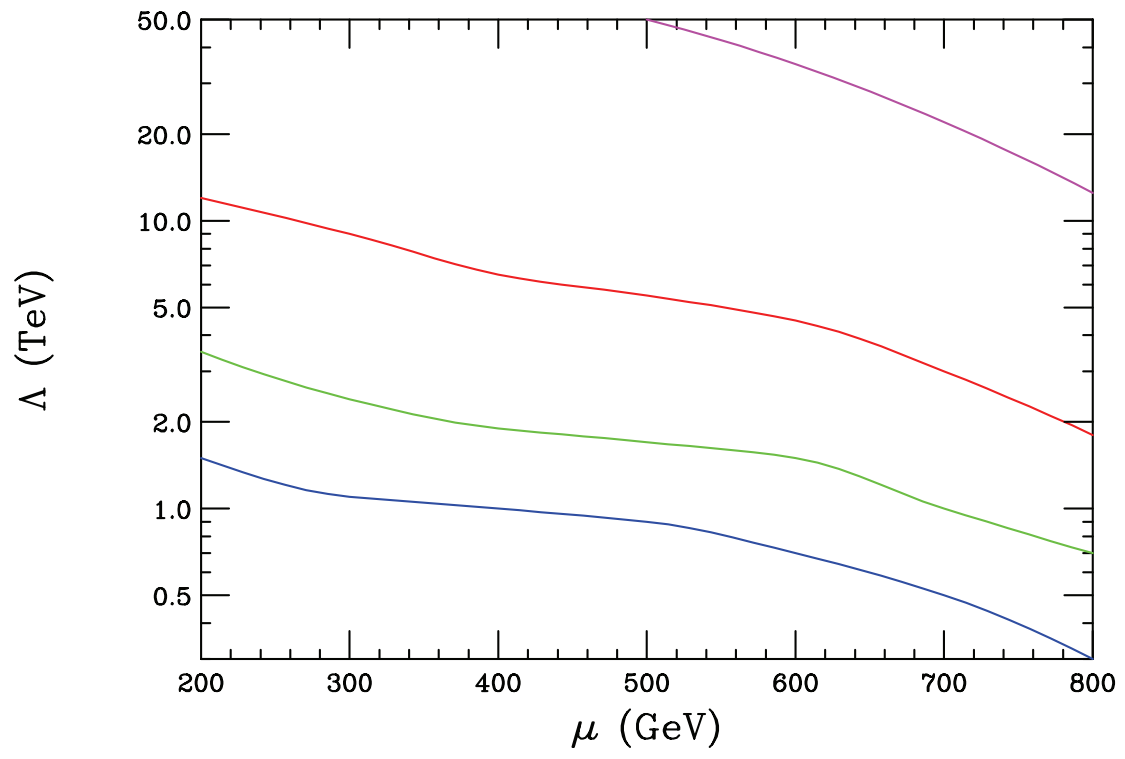

Figure 4: Approximate lower bounds of the scale $\Lambda$ as a function of $\mu$ from the Tevatron. From top to bottom the curves correspond to $d=1.4,1.5,1.6$ and 1.7 , respectively

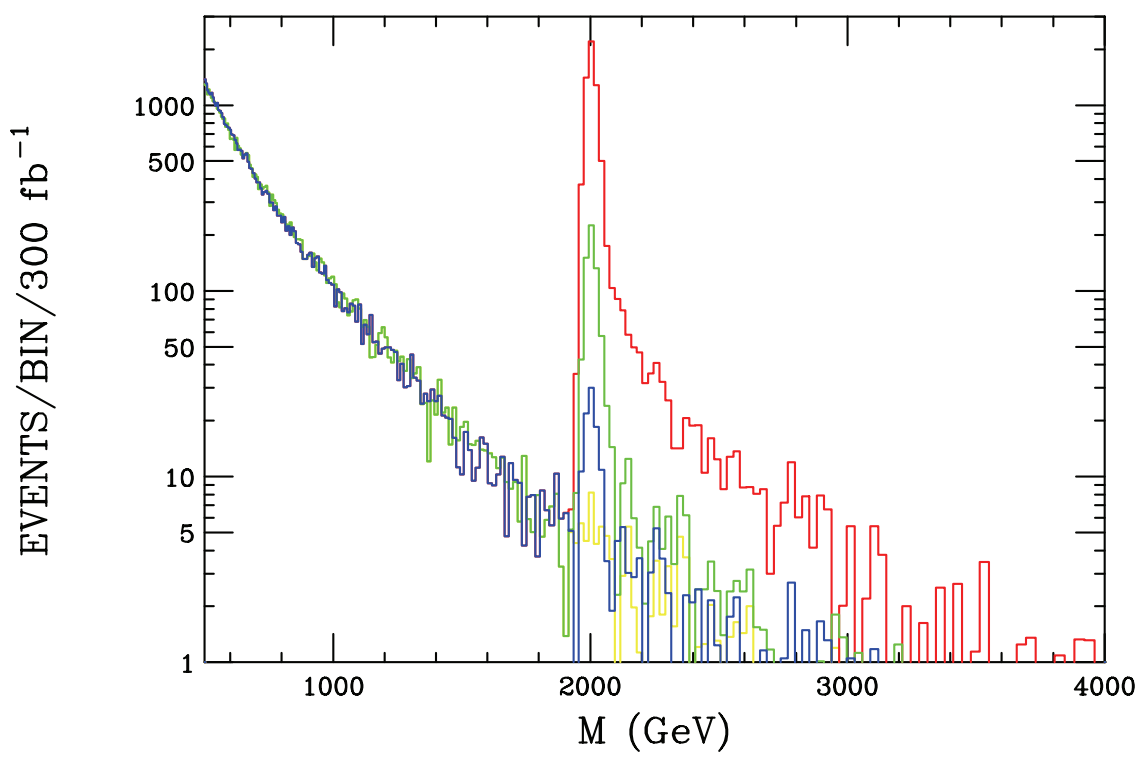

Figure 5: High resolution scan of the Drell-Yan dilepton mass distribution at the LHC assuming a large integrated luminosity taking $d=1.5, \mu=2 \mathrm{TeV}$ with $\Lambda=3(10,25) \mathrm{TeV}$ corresponding to the red(green,blue) histogram. The yellow histogram is the SM prediction. Adding the contribution from the $g g$ initial state makes an invisible contribution to this result. 
of any kind by the Tevatron clearly already constrains these model parameters. Certainly if $\mu$ is sufficiently small such an unresonance structure would have been already observed[30] unless $\Lambda$ was simultaneously also very large. For example, a simple analysis indicates that if $d=1.5$ then we must have $\mu>600 \mathrm{GeV}$ unless $\Lambda \gtrsim 4.5 \mathrm{TeV}$. For smaller values of $\Lambda$, somewhat larger values of $\mu$ can be excluded. Furthermore, these constraints are seen to become stronger(weaker) for smaller(larger) values of $d$. A rough summary of the possible Tevatron constraints on the model parameters from the lack of observation of any Drell-Yan resonances is shown in Fig. 4. It is interesting to note that if such an unresonance were to become visible at the Tevatron in the near future it would be somewhat difficult to distinguish it from a more ordinary resonance due to the low statistics available. It goes without saying that a detailed determination of the model parameters from such a resonance at the Tevatron would also be difficult if not impossible. As an aside, it is interesting to note that such an unresonance should also have appeared at LEP II in all final state fermion channels provided $\Lambda$ is not too large, $\lesssim 5-10 \mathrm{TeV}$, and this also suggests that $\mu \gtrsim 200 \mathrm{GeV}$ since the effective mass resolution of LEP detectors was somewhat better than those at the Tevatron.

Let us now look ahead to the case of LHC where for simplicity we will restrict our attention to cases where $\mu \geq 1 \mathrm{TeV} . \S$ To get a first idea of what these unparticle thresholds may look like in detail we show in Fig. 5 a simple example assuming that $d=1.5$ and $\mu=2 \mathrm{TeV}$ with a large integrated luminosity and a fine-grained binning comparable to the dilepton mass resolution. Unlike the Tevatron, where the unresonance peak does an excellent job at faking a more typical resonance structure, at the LHC we see that there is instead an abrupt cross section edge at $M \simeq \mu$ followed by a substantial tail at larger values of $M$. In this first sample result we find that such a bizarre structure will be observable even for $\Lambda$ values in excess of $25 \mathrm{TeV}$ although the identification of its special nature is lost in this large $\Lambda$ limit due to low statistics. For more modest values of $\Lambda$ the cross section rise is still by more than an order of magnitude indicating that this unusual new physics will be difficult to miss in this particular channel.

\footnotetext{
${ }^{\S}$ We note that smaller values of $\mu$ with significantly larger values of $\Lambda$ remain allowed by both the Tevatron and LEP II data discussed above.
} 

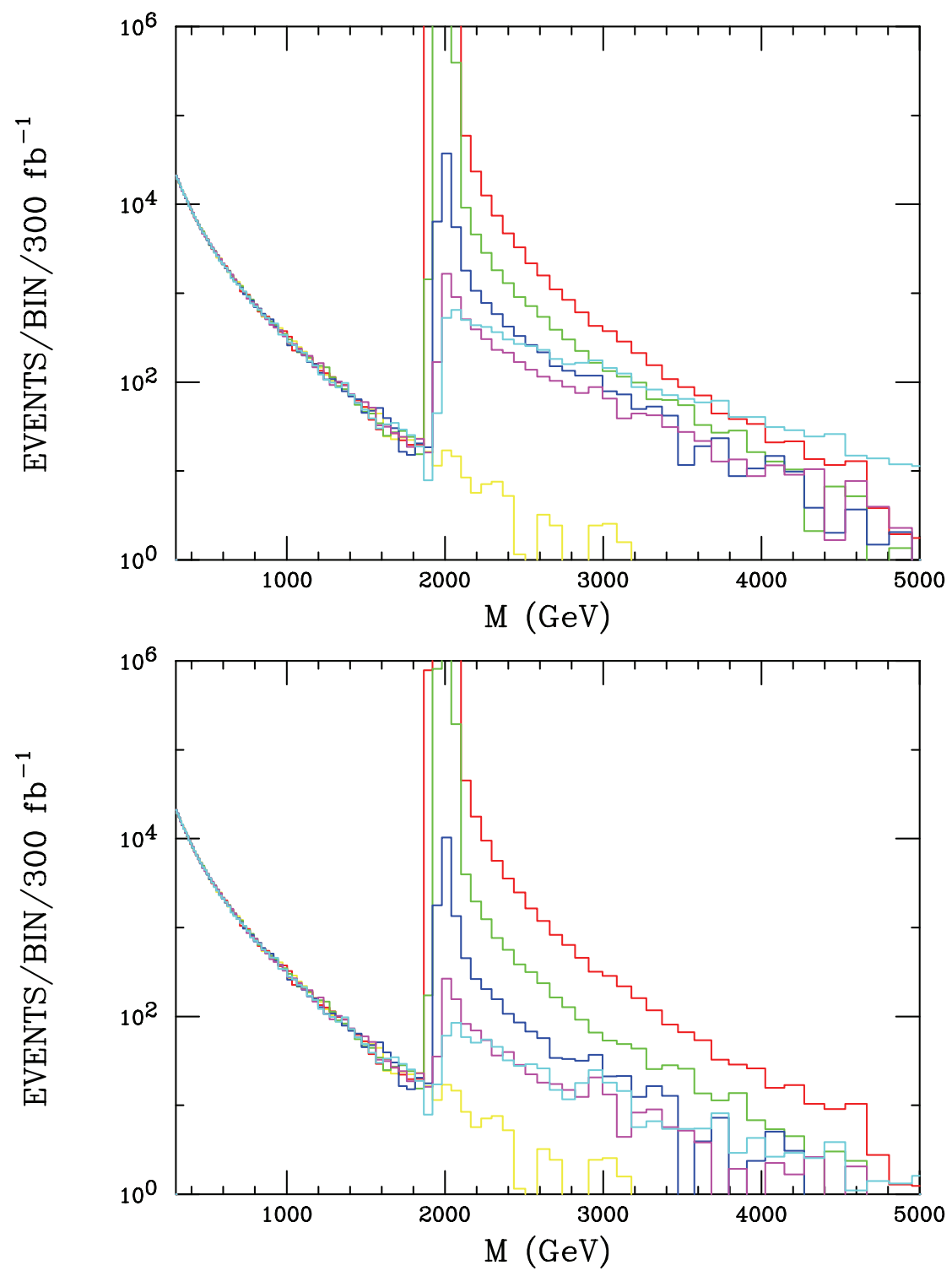

Figure 6: Drell-Yan dilepton mass distribution at the LHC with high luminosity assuming $\mu=2$ $\mathrm{TeV}$ with $\Lambda=1(2) \mathrm{TeV}$ in the top(bottom) panel. The yellow histogram is the SM prediction while the red(green,blue,magenta,cyan) histograms corresponds to $d=1.1(1.3,1.5,1.7,1.9)$, respectively. 

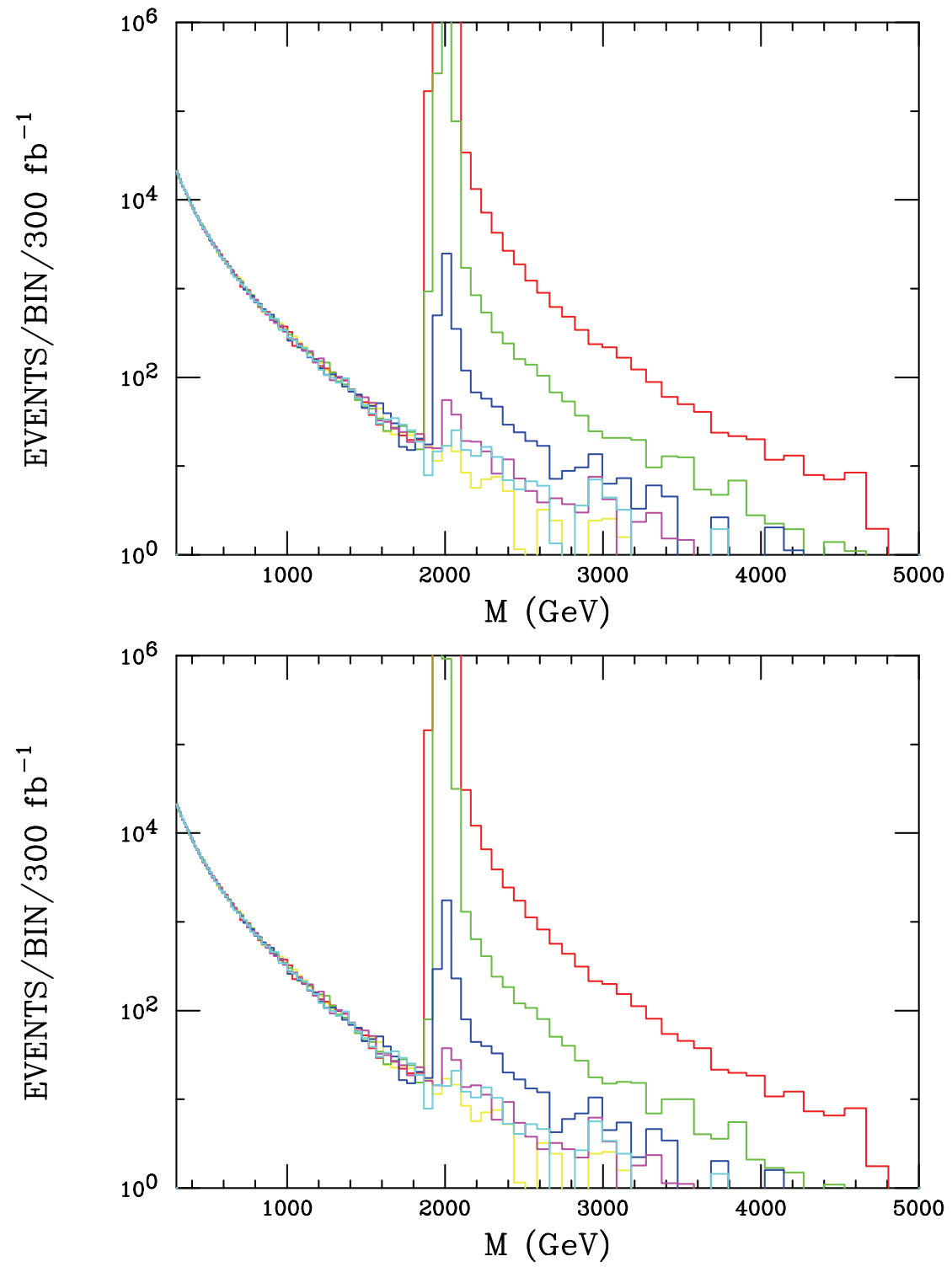

Figure 7: Same as in the previous figure but now with $\Lambda=3(4) \mathrm{TeV}$ in the top(lower) panel. 
Figs. 6,7,8 and 9 show an array of unparticle model predictions for the LHC assuming a high integrated luminosity as the three parameters are varied independently. Several things are immediately clear from these results: $(i)$ As expected, the abrupt increase in the cross section due to the unresonance allows for a rather precise determination of the value of $\mu$ over a very large range of the other parameters. (ii) The specific details of the height of the peak and the shape of the tail of the unresonance structure itself is controlled by a combination of the both the $d$ and $\Lambda$ parameters. The height of the peak is found to be only weakly dependent on the value of $\Lambda$ itself when the corresponding value of $d$ is small, $\gtrsim 1$. However, this sensitivity grows rapidly as $d$ is increased and is found to scale as $\sim 1 / \Lambda^{2(d-1)}$ for fixed $d$. On the otherhand, for fixed $\Lambda$, the unresonance peak height is expected to scale exponentially with $d$ which explains the strong $d$ dependence we observe in these figures. Note that the generic shape of the high energy tail of the distribution undergoes a significant qualitative change as the value of $d$ passes through $d=1.5$. Provided a signal of the unresonance is indeed observed at the LHC with sufficient statistics it would seem from these figures that it will be easily distinguished from a more traditional $Z^{\prime}$-like resonance. Furthermore, it would also appear to be quite straightforward to extract the values of the parameters $\mu, d$ and $\Lambda$ with reasonable precision from the location and height of the peak itself as well as the shape of its high energy tail.

One can also ask what ranges of the parameter space of the unparticle scenario can be explored at the LHC with high luminosity; the answer lies in Fig. 10. Here we see that, e.g., with $\mu=2 \mathrm{TeV}$ and $d=1.5$, the LHC has significant sensitivity to unresonance production up to values of $\Lambda$ in the 30-40 $\mathrm{TeV}$ range. As expected this sensitivity is seen to decline(improve) as either $\mu$ or $d$ is increased(decreased). Of course, with a lower integrated luminosity the reach will not be as great but will still remain significantly better than that obtainable at the Tevatron.

In this paper we have explored the physics associated with spin-1 unparticle exchange in the Drell-Yan process, $g g, q \bar{q} \rightarrow \ell^{+} \ell^{-}$, at both the Tevatron and the LHC. The new physics associated with these uunparticles can be truly bizarre. The analysis presented here was based 

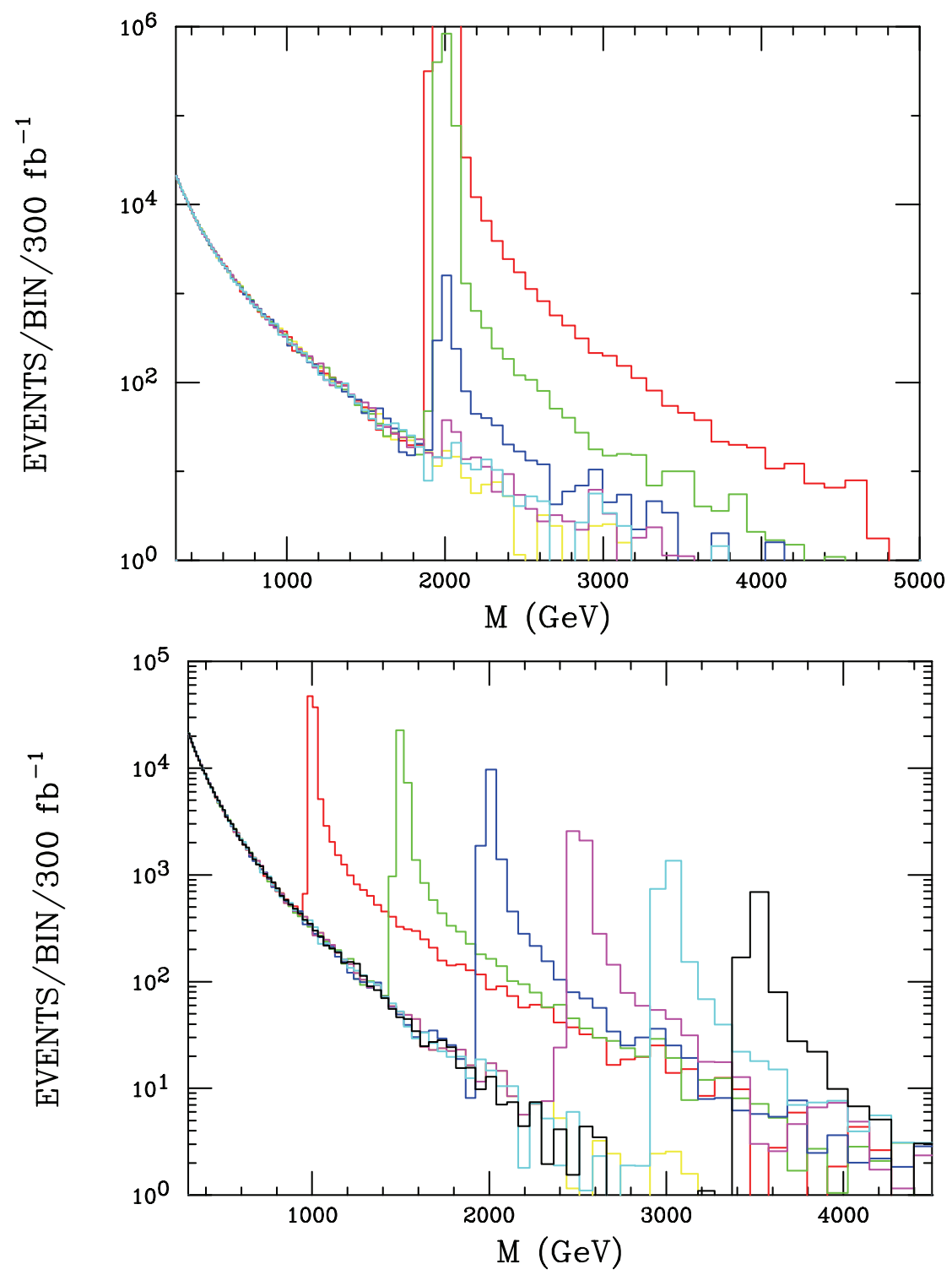

Figure 8: In the top panel, same as the previous figure but now with $\Lambda=5 \mathrm{TeV}$. In the lower panel, $d=1.5$ and $\Lambda=2 \mathrm{TeV}$ is assumed for the signal histograms which, from left to right, correspond to $\mu=1,1.5,2,2.5,3$ and $3.5 \mathrm{TeV}$, respectively. 

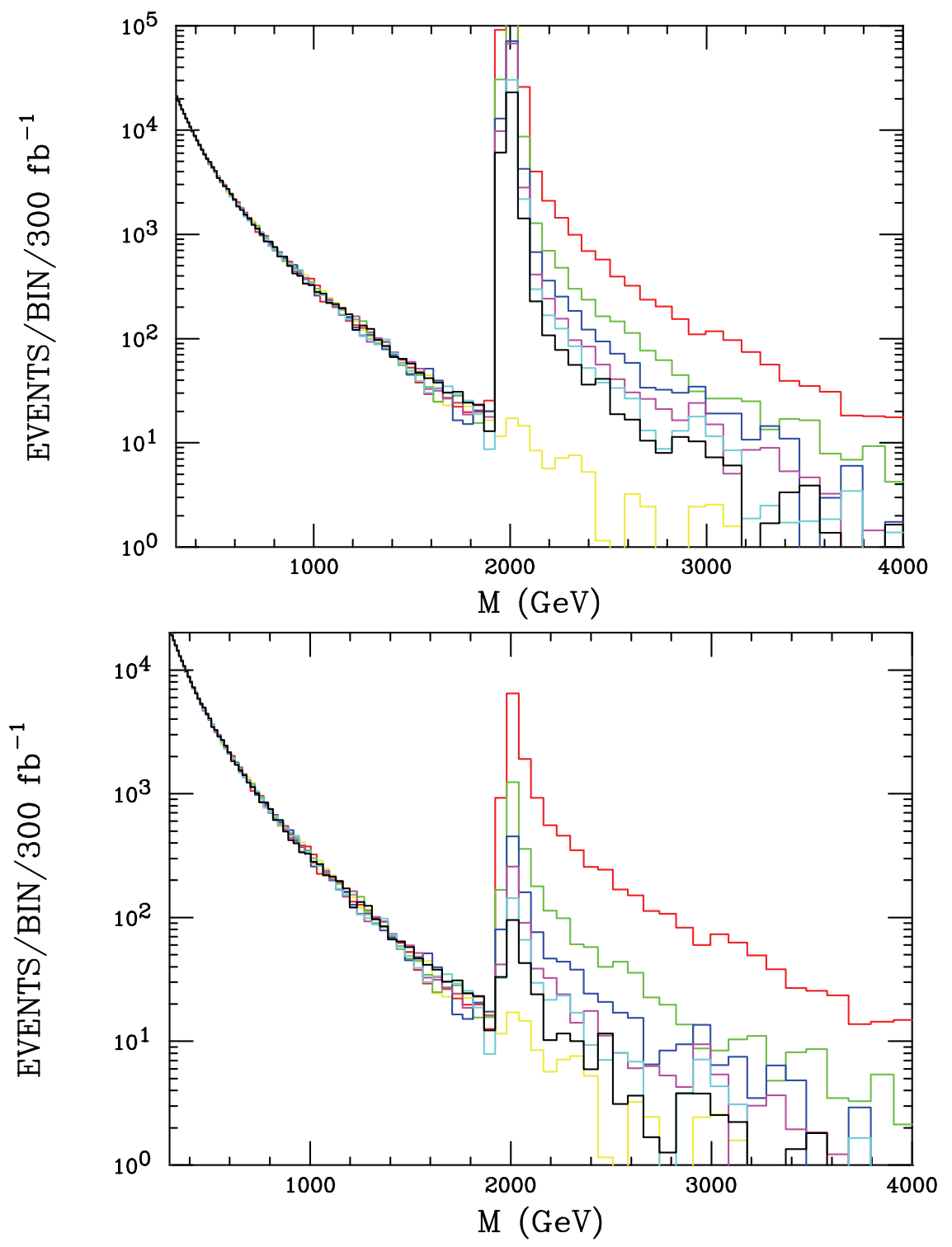

Figure 9: Same as in Fig. 6 but now with $d=1.4(1.6)$ and $\mu=2 \mathrm{TeV}$ in the top(bottom) panel. The signal histograms, from top to bottom, correspond to $\Lambda=1-6 \mathrm{TeV}$ in steps of $1 \mathrm{TeV}$. 


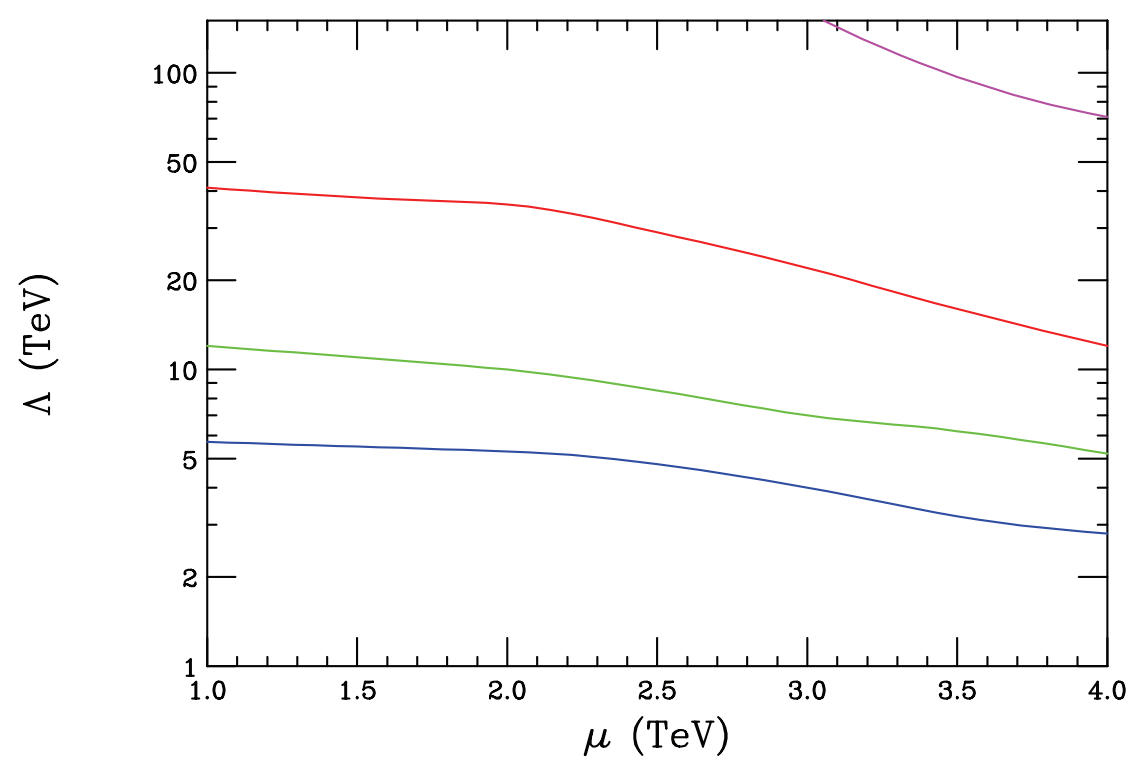

Figure 10: $5 \sigma$ search reach as in Fig. 4 but now for the LHC assuming an integrated luminosity of $300 \mathrm{fb}^{-1}$.

on two assumptions: $(i)$ that the unparticle couples universally to all SM fermion representations and $(i i)$ the propagator for the unparticle due to the Higgs-induced broken conformal symmetry was at least approximately that given in Ref.[15]. In this picture, all of the very strong low-energy constraints on unparticle exchanges are naturally avoided and the relevant scale $\Lambda$ may be as low as a few $\mathrm{TeV}$ and hence accessible to colliders. In our analysis we primarily focused on the preferred parameter space region $1 \leq d \leq 2$. In this case the form of the unparticle propagator leads to a sharp threshold in the contribution of unparticles to all exchange processes including Drell-Yan. For $d$ in this range this structure appears pseudoresonance-like, whose location is determined by the propagator parameter $\mu$, which we term an unresonance. With low statistics the unresonance would appear to be a typical narrow resonance as one would imagine being produced by a weakly interacting $Z^{\prime}$ state. However, the true nature of this structure was shown here to be quite distinct and detectable over a wide range of model parameters at both the Tevatron and the LHC given sufficient luminosity. Furthermore, the shape of the unresonance structure and its tail was shown to be sufficiently sensitive to the parameters $d$ and $\Lambda$ that their values could be determined from the data. 
Due to the, at least approximate, $\theta$-function turn on of the effects of unparticle exchanges, the new physics associated with such particles is not detectable at energy scales below $\mu$; this is quite unlike the exchanges of more ordinary massive particles whose effects may be detected indirectly below threshold. This implies that the physics of unresonances will not be accessible at the ILC if $\mu>1 \mathrm{TeV}$.

It would be extremely interesting if such unphysics were to make its appearance at the LHC.

\section{Acknowledgments}

The author would like to thank JoAnne Hewett for discussions related to this paper.

\section{References}

[1] H. Georgi, arXiv:hep-ph/0703260.

[2] T. Banks and A. Zaks, Nucl. Phys. B 196, 189 (1982).

[3] H. Georgi, arXiv:0704.2457 [hep-ph].

[4] K. Cheung, W. Y. Keung and T. C. Yuan, arXiv:0704.2588 [hep-ph].

[5] M. Luo and G. Zhu, arXiv:0704.3532 [hep-ph].

[6] C. H. Chen and C. Q. Geng, arXiv:0705.0689 [hep-ph].

[7] G. J. Ding and M. L. Yan, arXiv:0705.0794 [hep-ph].

[8] Y. Liao, arXiv:0705.0837 [hep-ph].

[9] T. M. Aliev, A. S. Cornell and N. Gaur, arXiv:0705.1326 [hep-ph].

[10] S. Catterall and F. Sannino, arXiv:0705.1664 [hep-lat]. 
[11] X. Q. Li and Z. T. Wei, arXiv:0705.1821 [hep-ph].

[12] M. Duraisamy, arXiv:0705.2622 [hep-ph].

[13] C. D. Lu, W. Wang and Y. M. Wang, arXiv:0705.2909 [hep-ph].

[14] M. A. Stephanov, arXiv:0705.3049 [hep-ph].

[15] P. J. Fox, A. Rajaraman and Y. Shirman, arXiv:0705.3092 [hep-ph].

[16] N. Greiner, arXiv:0705.3518 [hep-ph].

[17] H. Davoudiasl, arXiv:0705.3636 [hep-ph].

[18] D. Choudhury, D. K. Ghosh and Mamta, arXiv:0705.3637 [hep-ph].

[19] S. L. Chen and X. G. He, arXiv:0705.3946 [hep-ph].

[20] T. M. Aliev, A. S. Cornell and N. Gaur, arXiv:0705.4542 [hep-ph].

[21] P. Mathews and V. Ravindran, arXiv:0705.4599 [hep-ph].

[22] S. Zhou, arXiv:0706.0302 [hep-ph].

[23] G. J. Ding and M. L. Yan, arXiv:0706.0325 [hep-ph].

[24] C. H. Chen and C. Q. Geng, arXiv:0706.0850 [hep-ph].

[25] M. Bander, J.L. Feng, A. Rajaraman, and Y. Shirman, arXiv:0706.2677 [hep-ph].

[26] For some background and basic formalism, see, T. G. Rizzo, arXiv:hep-ph/0610104.

[27] T. Kamon, M. G. Albrow et al. [CDF Collaboration], Nucl. Instrum. Meth. A 480, 524 (2002).

[28] CMS Physics TDR, Volume II: CERN-LHCC-2006-021, ATLAS TDR, http://atlas.web.cern.ch/Atlas/GROUPS/PHYSICS/TDR/TDR.html. 
[29] E. Eichten, K. D. Lane and M. E. Peskin, Phys. Rev. Lett. 50, 811 (1983).

[30] The direct search lower limit on , e.g., the mass of a new $Z^{\prime}$ gauge boson with SM couplings is approaching $1 \mathrm{TeV}$ from Run II data at the Tevatron. The lower bound on a SM-coupled $Z^{\prime}$ is $923 \mathrm{GeV}$ from CDF. See, for example, P. Savard, "Searches for Extra Dimensions and New Gauge Bosons at the Tevatron," talk given at the XXXIII International Conference on High Energy Physics, 26 July-2 August 2006, Moscow, Russia; T. Adams, "Searches for New Phenomena with Lepton Final States at the Tevatron," talk given at Rencontres de Moriond Electroweak Interactions and Unified Theories 2007, La Thuile, Italy 10-17 March 2007. 